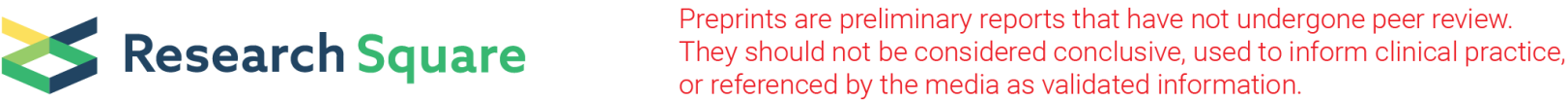

\section{Diversity of human rhinovirus species among children with severe or mild acute lower respiratory infection in Beijing, China, during 2016-2019}

\section{Dongmei Chen}

Capital Institute of Pediatrics

\section{Ruoxi Zhang}

National Institute of Securities Markets

\section{Yuan Qian}

Capital Institute of Pediatrics

\section{Yu Sun}

Capital Institute of Pediatrics

\section{Runan Zhu}

Capital Institute of Pediatrics

\section{Fang Wang}

Capital Institute of Pediatrics

\section{Yaxin Ding}

Capital Institute of Pediatrics

\section{Yutong Zhou}

Capital Institute of Pediatrics

\section{Qi Guo}

Capital Institute of Pediatrics

\section{Dong Qu}

Affilated Children's Hospital of Capital Institute of Pediatrics

\section{Ling Cao}

Affilated Children's Hospital of Capital Institute of Pediatrics

Linqing Zhao ( $\square$ linqingz525@163.com )

Capital Institute of Pediatrics https://orcid.org/0000-0002-5622-1959

\section{Research article}

Keywords: Pediatric patients, acute lower respiratory infection, human rhinovirus, species, diversity

Posted Date: November 1st, 2019

DOI: https://doi.org/10.21203/rs.2.16727/v1 
License: (c) (i) This work is licensed under a Creative Commons Attribution 4.0 International License. Read Full License 


\section{Abstract}

Background Human rhinovirus (HRV) infections were confirmed in severe acute lower respiratory infections (ALRI). Methods To evaluate the diversity and circulation pattern of HRVs species, specimens were collected from pediatric patients with ALRI during Dec 2016 to Feb 2019 and screened by RVP FAST Assay. Specimens positive for HRVs/ Enteroviruses (EVs) were then tested for HRVs and EVs by PCR. Then the capsid protein gene of HRVs was amplified and sequenced for species identification, and clinical data of HRV infections were analyzed. The chi-square $(\chi 2)$ test and rank sum test was used for statistical analysis using SPSS Statistics 22.0 version. In order to test and evaluate the relationship between patients positive for different HRV species and the outcome of Intensive Care Unit (ICU) (ICU group) or Department of Respiratory admission (Respiratory group), a logistic model was constructed using multiple logistic regression analysis. Results Among 1835 specimens tested, 363 (19.8\%) were positive for EVs/HRVs, and 314 (86.5\%, 314/363) were positive for HRVs, including 177 (56.4\%, 177/314) HRV A, 29 (9.2\%, 29/314) HRV B, and 108 (34.4\%, 108/314) HRV C. Using HRV C as the control species, HRV A was a dangerous factor for severe clinical outcome (OR=1.983, 95\% Cl=1.091-3.605) ( $p=0.025)$. Patients positive for HRVs from ICU group were significantly younger than those from Respiratory group ( 0.39 years: 1.80 years, $p=0.000$ ) and age was confirmed as a protective factor for severe ALRI. In August and September, HRV A and B are the dominant species, then HRV C in October and December. Among 96 types confirmed in the study, the predominant types usually showed in no consecutive years. Conclusions HRVs were important viral pathogens for ALRI in children. HRV A and C are more popular. Compared to HRV C, HRV A were associated more powerful with severe ALRI. The risk of severe ALRI by HRVs infections is decreased with the increasing of age.

\section{Introduction}

Human rhinovirus (HRV), first identified in 1956, had been noted as a major pathogen of common cold for over 50 years(1). With the development of molecular techniques for detecting HRVs in a variety of clinical specimens and the discovery of the genetically distinct HRV C species viruses, HRVs infections were confirmed not only in common colds, but also in otitis, sinusitis, severe lower respiratory infections, especially infantile bronchiolitis, childhood pneumonia, and acute exacerbations of chronic respiratory diseases, such as asthma $(2,3)$.

Based on the nucleotide sequence homology, HRVs can be divided into three genetically distinct species: HRV A, HRV B and HRV C (4-6). It has been shown in a number of studies that HRV A and HRV C were associated with more severe outcome in the pediatric population (7-12). Other results supported that HRV C, which may be one of the key factors in the association of HRVs with asthma (13), may cause more severe illness, and is more frequently associated with wheezing episodes, asthma exacerbations, and lower respiratory tract infections than HRV A and HRV B $(8,14-16)$. However, data on the relationship between HRV species and severity of disease and the circulation patterns of HRVs still need to be accumulated. 
There are approximately 169 serotypes or genotypes for the highly genetically and antigenically diverse of HRVs, which compound the difficulties for pan-HRV detection in a universal nucleotide test. Therefore, the $5^{\prime}$ untranslated region (5' UTR) of HRVs genome containing several highly conserved regions is the common target for primer design $(4,17,18)$. For they share greater than $70 \%$ amino acid identity in the P1 $2 C$, and $3 C D$ regions with other members of the Enterovirus genus, HRVs, with small, non-enveloped, positive-stranded RNAs, were assigned to the Enterovirus genus within the Picornaviridae family. However, sequences in the capsid protein coding region are more variable, which helps to distinguish genotypes (5).

To evaluate the diversity of HRVs species among children with severe or mild lower acute respiratory infection in Beijing and to reveal the circulation patterns of HRVs, clinical specimens for respiratory virus screening were collected from pediatric patients during Dec 2016 to Feb 2019 for pan-HRV detection in a universal nucleotide test in the study, and then the capsid protein gene of HRVs was sequenced for species identification and clinical data were analyzed.

\section{Materials And Methods}

\section{Clinical specimens}

In the retrospective study, nasopharyngeal aspirates (NPA) were obtained for respiratory virus screening from patients aged $<16$ years who visited the Children's Hospital affiliated with the Capital Institute of Pediatrics (CIP) (Beijing, China) and were diagnosed with acute lower respiratory infections (ALRIs), including bronchitis, bronchiolitis, and pneumonia, between November 2016 to February 2019. The diagnosis of ALRI in this study was according to the Zhu Futang Textbook of Pediatrics (7 $7^{\text {th }}$ Edition) (19).

Specimens were immediately stored at $4^{\circ} \mathrm{C}$ and sent to the laboratory within 12 hours. Upon arrival at the laboratory, each of the clinical specimens was handled in a Class II bio-safety cabinet (BSC II) and processed immediately in $2.5 \mathrm{ml}$ of viral transport medium (VTM) (Yocon Biotechnology Co., Ltd, Beijing, China) and centrifuged at $500 \times \mathrm{g}$ for $10 \mathrm{~min}$ to obtain the supernatant for respiratory virus screening.

The study was approved by the Ethics Committee of the Capital Institute of Pediatrics.

\section{RVP FAST assay}

Total nucleic acid (DNA and RNA) was extracted from $200 \mu \mathrm{L}$ of each collected specimen using the QIAamp MinElute Virus Spin Kit (Qiagen $\mathrm{GmbH}$, Germany) according to the manufacturer's instructions. MS-2 bacteriophage was used as an internal control. Extracts were then tested for influenza A virus (Flu A) (with additional subtyping for $\mathrm{H} 1, \mathrm{H} 3$, and $\mathrm{H} 5$ ) and Flu $\mathrm{B}$, respiratory syncytial virus (RSV) A and RSV B, parainfluenza virus 1-4 (PIV1-4), human adenovirus (HAdV), human metapneumovirus (HMPV), coronaviruses 229E, NL63, OC43, and HKU1, enterovirus (EVs)/HRVs, and human bocavirus (HBoV) using the RVP FAST assay (Luminex Molecular Diagnostics Inc., Toronto, Canada) according to the manufacturer's instructions in a 96-well plate format. The plate was then analyzed using a Luminex 200 
(Luminex Corporation, USA). And the data were analyzed with the TDAS RVP Fast of XTAG Data Analysis Software (Luminex Molecular Diagnostics Inc., Canada). A mean fluorescence intensity (MFI) value above the threshold level for a particular target indicated a positive result for that target.

\section{Identification and genotyping of HRVs or EVs by RT-PCR and sequence analysis}

For EVs/HRVs-positive clinical specimens, semi-nested revere-transcription PCR (RT-PCR) was performed for HRV-confirmation, and the amplified products of the second semi-nested PCR for a 539bp nucleotides fragment within the VP4/VP2 region were sequenced ${ }^{[13]}$. For HRVs-negative specimens, another RT-PCR was subsequently performed for EVs screening, and the amplified products were then sequenced (20).

PCR products were sequenced by Sino Geno Max Co., Ltd., Beijing, China. The obtained sequences were verified by using NCBI BLAST (http://blast.ncbi.nlm.nih.gov/). Phylogenetic analyses conducted with the MEGA version 6.0 software were used to identify the HRV or EV species, as well as to construct the phylogenetic trees using the neighbor-joining method and maximum composite likelihood model with bootstrap values estimated with 1000 bootstrap replications, assessing the reliability of individual nodes in each phylogenetic tree (21).

\section{Clinical data collection}

The medical records of pediatric patients with confirmed HRV species were reviewed. The following clinical data were extracted: age, gender, length of stay in hospital, the departments discharged from hospital, date of sample collection, clinical diagnosis, hospitalization status, and laboratory values.

\section{Statistical analysis}

The age of rhinovirus-positive patients were described using the median and interquartile range. The chisquare $\left(\mathrm{X}^{2}\right)$ test and rank sum test was used for statistical analysis using SPSS Statistics 22.0 version (IBM, NY, USA). The $p$ values were considered significant under the 0.05 level. In order to test and evaluate the relationship between patients positive for different HRV species and the outcome of Intensive Care Unit (ICU) (ICU group) or Department of Respiratory admission (Respiratory group), a logistic model was constructed using multiple logistic regression analysis.

\section{Results}

\section{General results of respiratory virus screening using RVP FAST assay}

During Nov 2016 to Feb 2019, 2090 respiratory specimens were collected for respiratory virus screening using RVP FAST assay. For there were more specimens belonging to one patient in one time hospitalization, we only kept the first or the EVs/HRVs positive one. Then 1835 specimens were left for statistical analysis. Among these specimens, there were $402(21.9 \%, 402 / 1835)$ for RSV, including 300 for RSV A and 114 for RSV B; 363 (19.8\%, 363/1835) positive for EVs/HRVs; 196 (10.7\%, 196/1835) for PIVs, including 27 for PIV1, 8 for PIV2, 136 for PIV3, and 30 for PIV4; 173 (9.4\%, 173/1835) for HBoV; 154 
(8.4\%, 154/1835) for ADV; 140 (7.6\%, 140/1835) for Flu, including 121 for Flu A (H1 91, H3 24), and 23 for Flu B; 80 (4.4\%, 80/1835) for HMPV; 60 for Coronavirus $(3.3 \%, 60 / 1835)$, including 29 for NL63, 6 for HKU1, 5 for 229E, and 21 for OC43.

\section{Identification and genotyping of HRVs or EVs}

For HRVs and EVs cannot be distinguished from each other in RVP FAST assay, RT-PCR and sequence analysis were used. Among 363 specimens positive for EVs/HRVs, 314 (86.5\%, 314/363) were determined as HRVs positive, including 177 (56.4\%, 177/314) positive for HRV A, 29 (9.2\%, 29/314) for HRV B, and $108(34.4 \%, 108 / 314)$ for HRV C, 19 (5.2\%, 19/363) were determined as EVs positive, and 30 were undetermined for low amplification products (Fig 1).

\section{The epidemiology of HRVs}

The monthly distribution of HRVs positive specimens during Nov 2016 to Feb 2019 was shown in Fig 2. HRV infections were shown all year round. More HRVs were detected through all Autumn months followed by that in Winter months. In August and September, HRV A and B are the dominant species, then HRV C became the dominant species in October and December.

By MEGA version 6.0 software, the phylogenetic tree shown in Fig 3 was constructed to identify the seroor geno-types of HRV positive specimens. Among 177 specimens positive for HRV A, 53 types were confirmed, including 15 positive for A49, 12 for A24, 10 for A12, and 9 for A101; Among 29 specimens positive for HRV B, 11 types were confirmed, including 8 for B79; Among 108 specimens positive for HRV C, 32 types were confirmed, including 14 for $\mathrm{C} 2,8$ for $\mathrm{C} 5$, and 6 for $\mathrm{C} 15$.

In Fig 4, no obvious circulation pattern was shown for each sero- or geno- type, therefore, more data should be accumulated to evaluate the epidemiology characters of HRV sero- or geno- types. For the 96 HRV types detected in the study, only 29 types were detected in consecutive years. The most prevalent strain of HRV A, A49 was found only between March 2017 and October 2017, which was followed by A24 more detected in the Autumn of 2018. The most prevalent strain of HRV B, B79 was detected in the winter of 2018. The most prevalent type of HRV C, C2 was detected more often in the late of 2018.

\section{Clinical characters of different HRV species in severe or mild acute lower respiratory tract infection}

As shown in Table 1, there were 925 patients discharged from Intensive Care Unit (ICU) with severe ALRI (ICU group) and 508 patients discharged from the Department of Respiratory with mild ALRI (Respiratory group) compared to those from ICU. The HRVs positive rates are similar and HRV A and HRV C are the major pathogens in two groups. However, the positive rates of HRVs species were significant different between the two groups $(p=0.011)$, which may be explained by more HRV A in ICU group (63.0\%: $45.2 \%$, $p=0.006)$, and more HRV B (15.1\%:6.5\%, $p=0.028)$ in Respiratory group. However, no significant difference was shown on HRV C (39.8\%: 30.5\%, $p=0.136)$ between the two groups. Compared to patients positive for HRVs from Respiratory group, patients who were positive for HRVs from ICU group were significantly younger ( 0.39 years: 1.80 years, $p=0.000$ ). 
In the ICU group, about $44.8 \%(n=69)$ were viral co-infection, with HBoV $(n=27)$ as the major virus followed by PIV ( $n=19)$ and RSV ( $n=18)$. In the Respiratory group, about $45.2 \%(n=42)$ were viral coinfection, with RSV as the most common viral pathogen $(n=12)$ followed by PIV $(n=11)$ and ADV $(n=9)$. No significant difference was shown in the whole viral co-infection $(p=0.957)$ and viral co-infection in different HRV species $(p=0.239)$ between ICU group and the Respiratory group.

Then a logistic model was constructed to test and verify the variables associated with severe ALRI (Table 2). By using HRV $C$ infection as the reference group among HRV species when gender, age and coinfections were considered as factors, there is a significant difference as the odds of HRV A related to severe ALRI was $\mathrm{OR}=1.983(95 \% \mathrm{Cl}=1.091-3.605)(p=0.025)$, while no significant difference was shown $(\mathrm{OR}=0.707,95 \% \mathrm{Cl}=0.260-1.920)(p=0.496)$ when the odds of HRV B related to severe ALRI was evaluated. In the logistic model, gender was excluded from variables associated with severe ALRI (OR=0.710, 95\% $\mathrm{Cl}=0.389-1.295, p=0.264)$, and age $(\mathrm{OR}=0.703,95 \% \mathrm{Cl}=0.607-0.813, p=0.000)$ was confirmed as a protective factor $(\mathrm{OR}<1$, the upper limit of $95 \% \mathrm{Cl}<1)$ for severe ALRI.

When the correlation of HRV sero- or geno- types with severe or mild ALRI was evaluated (Fig 5), 39 HRV types were shown in both groups. In ICU groups, HRV A24 followed by A49, HRV B79 followed by B6, and HRV C2 were the most popular serotypes of different species, while HRV A49 followed by A18, HRV B14, $\mathrm{B} 79$ and B92, and HRV C5 followed by C2 were the most popular serotypes of different species in Respiratory group. However, as a result of restrictions on the numbers of specimens belonging to each sero- or geno-type, no skewing towards one type in either group was observed.

\section{Discussion}

Determined by RVP FAST Assay in the study, EVs/HRVs were the second viral agent (19.8\%, 363/1835) following RSV $(21.9 \%, 402 / 1835)$ in ALRI during Nov 2016 to Feb 2019 in Beijing, and PIVs were the third one $(10.7 \%, 196 / 1835), \mathrm{HBoV}$, the fourth one $(9.4 \%, 173 / 1835)$, ADV, the fifth one (8.4\%, 154/1835), Flu, the sixth one $(7.6 \%, 140 / 1835), \mathrm{HMPV}$, the seventh one $(4.4 \%, 80 / 1835)$, Coronavirus, the eighth one $(3.3 \%, 60 / 1835)$. Zhao et al. has reported that viral infection was shown to be the most prevalent etiological agent among children with severe acute respiratory infection (SARIs) in either the Beijing or the Shanghai area, and RSV (52.9\%) and EVs/HRVs (34.7\%) were the most frequently detected respiratory virus in Beijing (22), which suggested that we should pay more attention to RSV and EVs/HRVs infection in children. In the study we described the epidemiology and clinical characters of HRV infections and analyzed the relationship between HRV species or sero-/geno-types and severity of clinical illness in young children to verify the variables associated with severe ALRI.

Among specimens positive for EVs/HRVs, only 5.2\% (19/363) were EVs positive, and nearly $90 \%$ (86.5\%, 314/363) were HRVs positive, which revealed that HRVs infection were weight highly over EVs infection in ALRI. We have reported in 2013 that HRVs are one of important pathogens for children with acute respiratory infection (ARI), especially ALRI in Beijing (23). In the study, HRV A $(56.4 \%, 177 / 314)$ was the predominant species in the study population, followed closely by HRV C $(34.4 \%, 108 / 314)$, then HRV B 
$(9.2 \%, 29 / 314)$, and the positive rates of each HRV species were significantly different between the two groups: ICU group with severe ALRI and Respiratory group with mild ALRI $(p=0.011<0.05)$, which may be supported by more HRV A in ICU group (63.0\%: 45.2\%, $p=0.006)$, and more HRV B (15.1\%: 6.5\%, $p=0.028)$ in Respiratory group. In Shanghai, China, HRV A (14.0\%) was the predominant species, followed by HRV C (5.6\%), then HRV B $(2.1 \%)$ in SARIs, too. However, their results showed no significant difference in clinical symptoms according to HRV species (14). In the study, when gender, age and co-infection were all considered and using HRV $C$ as the control species, HRV A was a dangerous factor to cause severe clinical outcome (OR=1.983>1,95\% Cl=1.091-3.605, the lower limit of $95 \% \mathrm{Cl}>1)(p=0.025)$, and HRV B has no relationship with severity of clinical outcome. Therefore, infection of HRV A was weight higher than that of HRV C in causing severe ALRI. Patients who were positive for HRVs from ICU group were significantly younger than those from Respiratory group ( 0.39 years: 1.80 years, $p=0.000)$, and age $(\mathrm{OR}=0.703,95 \% \mathrm{Cl}=0.607-0.813, \mathrm{p}=0.000)$ was confirmed as a protective factor (OR $<1$, the upper limit of $95 \% \mathrm{Cl} 1$ ) for severe ALRI, which implied that the risk of severe ALRI by HRVs infections is decreased with the increasing of age. In three Sub-Saharan African countries, those with pneumonia and HRV C were older (12.1 vs. 9.4 months, $P=0.033)$ and more likely to present with wheeze ( $35 \%$ vs. $25 \%, P=0.031)$ compared to HRV A cases (13). Therefore, age is an important variable associated with severe ALRI when HRVs infections were evaluated. It has been demonstrated that cellular receptor for the majority of HRV A and $B$ (major group) is intercellular adhesion molecule 1 (ICAM-1), for a minority of isolates (minor group, $\sim 10$ HRV A), low-density lipoprotein receptor (LDLR) family members (24), for HRV C, the cadherin-related family member 3 (CDHR3) suggested (25). These considerable diversities of genetic, immunogenic, and receptor use properties might account for the differences in clinical presentation of different HRV species infection (26).

Lau et al. reviewed that the seasonal circulation of HRVs varied geographically, which usually peaked in fall or winter in most temperate or subtropical countries(7). In Taiwan, HRV infections were predominant during fall and winter seasons (27). In the study, more HRVs were detected in Autumn months followed by that in Winter months. HRV A and B are the dominant species in August and September, while HRV C became the dominant species in October and December.

Among approximately 169 sero- or geno-types of HRVs reported, 98 were confirmed in the study, including 53 types of HRV A, 11 types of HRV B, 34 types of HRV C. In HRV A, A49 was the dominant type, in HRV B, B79, in HRV C, C2. However, HRV A24, B79, and C2 were the most popular serotypes of different species, respectively, in ICU groups, while HRV A49, B14, and C5 were the most popular serotypes of different species, respectively, in Respiratory group. In the research work, although no obvious circulation pattern was shown for each sero- or geno- type, most prevalent strains were not detected in consecutive years, for example, A49 was found only between March 2017 and October 2017, B79 was detected in the winter of 2018 , and $\mathrm{C} 2$ was detected more often in the late of 2018. We did not observe some skewing towards one type in children with severe illness. In Shanghai, 77 sero- or geno- types were detected including 43 for HRV A, 10 for HRV B, and 24 for HRV C, among which A78, A12, A89, B70, C2, C6, and C24 predominated during 2013-2015 (14). More data should be accumulated to reveal the epidemiology characters of each sero- or geno- type. 
There are some limitations in the study. Co-bacterial infection was not evaluated, which maybe a variable associated with the severity of ALRI in HRVs infections. We collected specimens only in two years, and more data should be accumulated to provide more powerful information supporting the circulation patterns of HRVs concluded in the study.

In conclusion, HRVs outweigh EVs and were second only to RSV as the important viral pathogens for ALRI in children in Beijing during 2016 to 2019. HRV A and C are more popular than HRV B in two groups with mild or sever ALRI. By using HRV $C$ as the reference species, HRV A were associated more powerful with severe ALRI, and age was a variable when association of HRV species infection with the severity of disease was evaluated. The predominant sero- or geno-types were different in two groups with mild or sever ALRI, and usually showed in no consecutive years.

\section{List Of Abbreviations}

Human rhinovirus (HRV); acute lower respiratory infections (ALRI); Enteroviruses (EVs); Intensive Care Unit (ICU); Capital Institute of Pediatrics (CIP); influenza A virus (Flu A); respiratory syncytial virus (RSV); parainfluenza virus 1-4 (PIV1-4); human adenovirus (HAdV); human metapneumovirus (HMPV); coronaviruses 229E, NL63, OC43, and HKU1; human bocavirus (HBoV); A mean fluorescence intensity (MFI); revere-transcription PCR (RT-PCR); the cadherin-related family member 3 (CDHR3); intercellular adhesion molecule 1 (ICAM-1); low-density lipoprotein receptor (LDLR).

\section{Declarations}

\section{Ethics approval:}

The study was approved by the Ethics Committee of the Capital Institute of Pediatrics.

\section{Consent for publication:}

Not applicable.

\section{Availability of data and materials:}

The datasets used and/or analysed during the current study are available from the corresponding author on reasonable request.

\section{Competing interests:}

The authors declare that they have no competing interests.

\section{Funding:}

This work was supported by grants from Beijing Municipal Commission of Health and Family (No. 2060399 PXM2017_026268_00005_00254486) and The Pediatric Medical Coordinated Development 
Center of Beijing Hospitals Authority (No. XTZD20180505).

\section{Authors' contributions:}

LZ conceived and designed the experiments. DC, $R(x) Z$, and $Y S$ performed the experiments. DC, $R(x) Z$, and $R(n) Z$ analyzed the data. $F W, Y D, Y Z, Q G, D Q$, and $L C$ contributed reagents/materials/analysis tools. $D C, R(x) Z$ and $L Z$ wrote the paper. $Y Q$ and $L Z$ reviewed the manuscript. $L Z$ is the critical reviewer for the manuscript and made final decision for the manuscript. All authors read and approved the final manuscript

\section{Acknowledgements}

Thanks for doctors' and nurses' devoting in clinical data collection. Thanks for pediatric patients' and their parents' consents for clinical specimens collection.

\section{References}

1. Price $W H$. The isolation of a new virus associated with respiratory clinical disease in humans. Proc Natl Acad Sci U S A. 1956; 42:892-6.

2. Louie JK, Roy-Burman A, Guardia-Labar L, Boston EJ, Kiang D, Padilla T, Yagi S, Messenger S, Petru AM, Glaser CA, Schnurr DP. Rhinovirus associated with severe lower respiratory tract infections in children. Pediatr Infect Dis J. 2009; 28:337-9.

3. Jartti T, Lehtinen P, Vuorinen T, Osterback R, van den Hoogen B, Osterhaus AD, Ruuskanen $O$. Respiratory picornaviruses and respiratory syncytial virus as causative agents of acute expiratory wheezing in children. Emerg Infect Dis. 2004; 10:1095-101.

4. Mclntyre CL, Knowles NJ, Simmonds P. Proposals for the classification of human rhinovirus species A, B and C into genotypically assigned types. J Gen Virol. 2013; 94:1791-806.

5. Wisdom A, Leitch EC, Gaunt E, Harvala H, Simmonds P. Screening respiratory samples for detection of human rhinoviruses (HRVs) and enteroviruses: comprehensive VP4-VP2 typing reveals high incidence and genetic diversity of HRV species C. J Clin Microbiol. 2009; 47:3958-67.

6. Savolainen C, Mulders MN, Hovi T. Phylogenetic analysis of rhinovirus isolates collected during successive epidemic seasons. Virus Res. 2002; 85:41-6.

7. Piralla A, Baldanti F, Gerna G. Phylogenetic patterns of human respiratory picornavirus species, including the newly identified group $C$ rhinoviruses, during a 1-year surveillance of a hospitalized patient population in Italy. J Clin Microbiol. 2011; 49:373-6.

8. Bizzintino J, Lee WM, Laing IA, Vang F, Pappas T, Zhang G, Martin AC, Khoo SK, Cox DW, Geelhoed GC, McMinn PC, Goldblatt J, Gern JE, Le Souef PN. Association between human rhinovirus C and severity of acute asthma in children. Eur Respir J. 2011; 37:1037-42.

9. Lau SK, Yip CC, Lin AW, Lee RA, So LY, Lau YL, Chan KH, Woo PC, Yuen KY. Clinical and molecular epidemiology of human rhinovirus $\mathrm{C}$ in children and adults in Hong Kong reveals a possible distinct 
human rhinovirus C subgroup. J Infect Dis. 2009; 200:1096-103.

10. Broberg E, Niemela J, Lahti E, Hyypia T, Ruuskanen O, Waris M. Human rhinovirus C-associated severe pneumonia in a neonate. J Clin Virol. 2011; 51:79-82.

11. Xiang Z, Gonzalez R, Xie Z, Xiao Y, Liu J, Chen L, Liu C, Zhang J, Ren L, Vernet G, Paranhos-Baccala G, Shen K, Jin Q, Wang J. Human rhinovirus $C$ infections mirror those of human rhinovirus $A$ in children with community-acquired pneumonia. J Clin Virol. 2010; 49: 94-99.

12. Louie JK, Yagi S, Nelson FA, Kiang D, Glaser CA, Rosenberg J, Cahill CK, Schnurr DP. Rhinovirus outbreak in a long term care facility for elderly persons associated with unusually high mortality. Clin Infect Dis. 2005; 41:262-5.

13. Zhao M, Zhu WJ, Qian Y, Sun Y, Zhu RN, Deng J, Wang F, Ding YX, Tian R, Liu CH, Meng LH, Zhao LQ. Association of Different Human Rhinovirus Species with Asthma in Children: A Preliminary Study. Chin Med J (Engl). 2016; 129:1513-8.

14. Zhao Y, Shen J, Wu B, Liu G, Lu R, Tan W. Genotypic diversity and epidemiology of human rhinovirus among children with severe acute respiratory tract infection in Shanghai, 2013-2015. Front Microbiol. 2018; 9:1836.

15. Baillie VL, Moore DP, Mathunjwa A, Morailane P, Simoes EAF, Madhi SA. Molecular subtyping of human rhinovirus in children from three sub-Saharan African countries. J Clin Microbiol. 2019; 57(9).

16. Gern JE. The ABCs of rhinoviruses, wheezing, and asthma. J Virol. 2010; 84:7418-7426.

17. Faux CE, Arden KE, Lambert SB, Nissen MD, Nolan TM, Chang AB, Sloots TP, Mackay IM. Usefulness of published PCR primers in detecting human rhinovirus infection. Emerg Infect Dis. 2011; 17:296298.

18. McLeish NJ, Witteveldt J, Clasper L, Mclntyre C, McWilliam Leitch EC, Hardie A, Bennett S, Gunson R, Carman WF, Feeney SA, Coyle PV, Vipond B, Muir P, Benschop K, Wolthers K, Waris M, Osterback R, Johannessen I, Templeton K, Harvala H, Simmonds P. Development and assay of RNA transcripts of enterovirus species $A$ to $D$, rhinovirus species a to $C$, and human parechovirus: assessment of assay sensitivity and specificity of real-time screening and typing methods. J Clin Microbiol. 2012; 50:29102917.

19. Hu Y, Jiang Z. Zhu Futang Textbook of Pediatrics, 7th ed ed. People's Medical Publishing House, Beijing. 2002.

20. Zhu RN, Qian Y, Deng J, Xing JF, Zhao LQ, Wang F, Liao B, Ren XX, Li Y, Zhang Q, Li J. [Study on the association of hand, foot and mouth disease and enterovirus 71/CA16 among children in Beijing, 2007]. Zhonghua Liu Xing Bing Xue Za Zhi. 2007; 28:1004-1008.

21. Tamura K, Stecher G, Peterson D, Filipski A, Kumar S. MEGA6: Molecular Evolutionary Genetics Analysis version 6.0. Mol Biol Evol. 2013; 30:2725-2729.

22. Zhao Y, Lu R, Shen J, Xie Z, Liu G, Tan W. Comparison of viral and epidemiological profiles of hospitalized children with severe acute respiratory infection in Beijing and Shanghai, China. BMC Infect Dis. 2019; 19:729. 
23. Song MH, Zhao LQ, Qian Y, Zhu RN, Deng J, Wang F, Sun Y, Tian R. [Different species of human rhinovirus infection in children with acute respiratory tract infections in Beijing]. Zhonghua Er Ke Za Zhi. 2013; 51:903-908.

24. Palmenberg AC, Gern JE. Classification and evolution of human rhinoviruses. Methods Mol Biol. 2015; 1221:1-10.

25. Palmenberg AC. Rhinovirus C, Asthma, and Cell Surface Expression of Virus Receptor CDHR3. J Virol. 2017; 91(7).

26. Cox DW, Le Souef PN. Rhinovirus and the developing lung. Paediatr Respir Rev. 2014; 15:268-274.

27. Hung HM, Yang SL, Chen CJ, Chiu CH, Kuo CY, Huang KA, Lin TY, Hsieh YC, Gong YN, Tsao KC, Huang YC. Molecular epidemiology and clinical features of rhinovirus infections among hospitalized patients in a medical center in Taiwan. J Microbiol Immunol Infect. 2019; 52:233-241.

\section{Tables}

Table 1. General characters of HRV detection in two groups: ICU group and Respiratory group

\begin{tabular}{|c|c|c|c|c|c|c|}
\hline Groups & No. tested, & No. positive for & No. positive for HRV & No. positive for HRV & No. positive for HRV & No. with viral co- \\
\hline & M (IQR) & HRVs (\%), M (IQR) & $\mathrm{A}(\%) *, \mathrm{M}(\mathrm{IQR})$ & $\mathrm{B}(\%)^{*}, \mathrm{M}(\mathrm{IQR})$ & $\mathrm{C}(\%)^{*}, \mathrm{M}(\mathrm{IQR})$ & infection (\%) \\
\hline \multirow[t]{2}{*}{ ICU } & $925,0.20$ & 154 (16.6), 0.39 ( & 97 (63.0), 0.34 (0.15- & 10 (6.5), 0.33 (0.21- & 47 (30.5), 0.64 (0.18- & $69(44.8)$ \\
\hline & $(0.11-0.76)$ & $0.15-1.05)$ & 0.96) & 1.92) & 1.09) & \\
\hline \multirow[t]{2}{*}{ Respiratory } & $508,2.62$ & 93 (18.3), 1.80 (0.84- & 42 (45.2), 1.50 (0.79- & 14 (15.1), 2.36 (1.05- & 37 (39.8), 1.84 (0.86- & $42(45.2)$ \\
\hline & $(1.00-5.34)$ & 3.82) & $3.80)$ & 7.11) & $3.74)$ & \\
\hline
\end{tabular}

M: Median age in year; (IQR: interquartile range; *Percent of No. positive for HRVs in the same group

Table 2. The data in the logistic model constructed to test and verify the variables associated with severe ALRI

\begin{tabular}{lccc}
\hline & OR Estimate & $95 \%$ CI & $p$ \\
\hline HRV A & 1.983 & $1.091-3.605$ & 0.025 \\
HRV B & 0.707 & $0.260-1.920$ & 0.496 \\
HRV C & Reference & - & 0.025 \\
Male gender & 0.710 & $0.389-1.295$ & 0.264 \\
Age & 0.703 & $0.607-0.813$ & 0.000 \\
Co-infection & 0.918 & $0.522-1.616$ & 0.768 \\
\hline
\end{tabular}


Figures

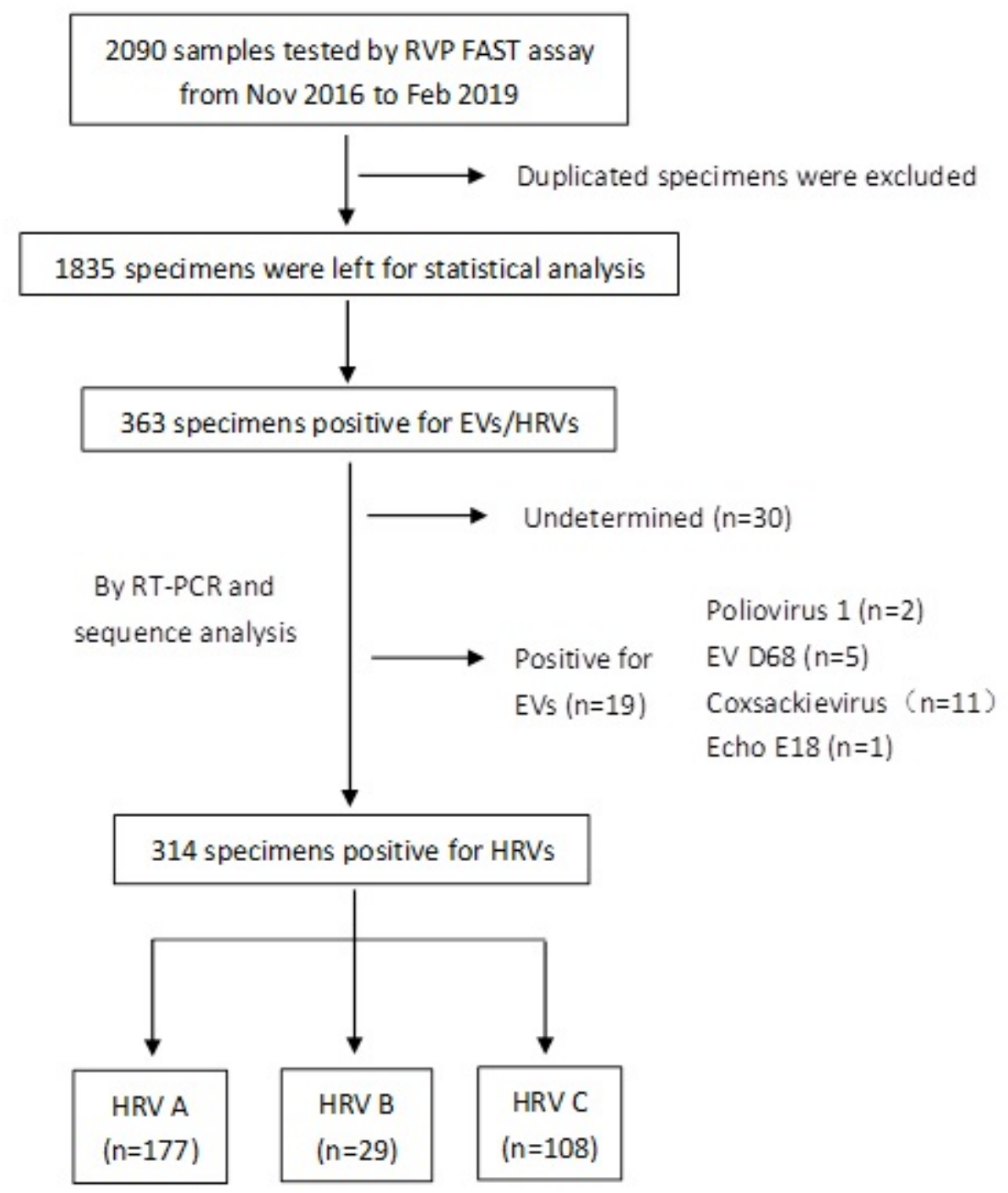

Figure 1

Scheme for specimens' selection 


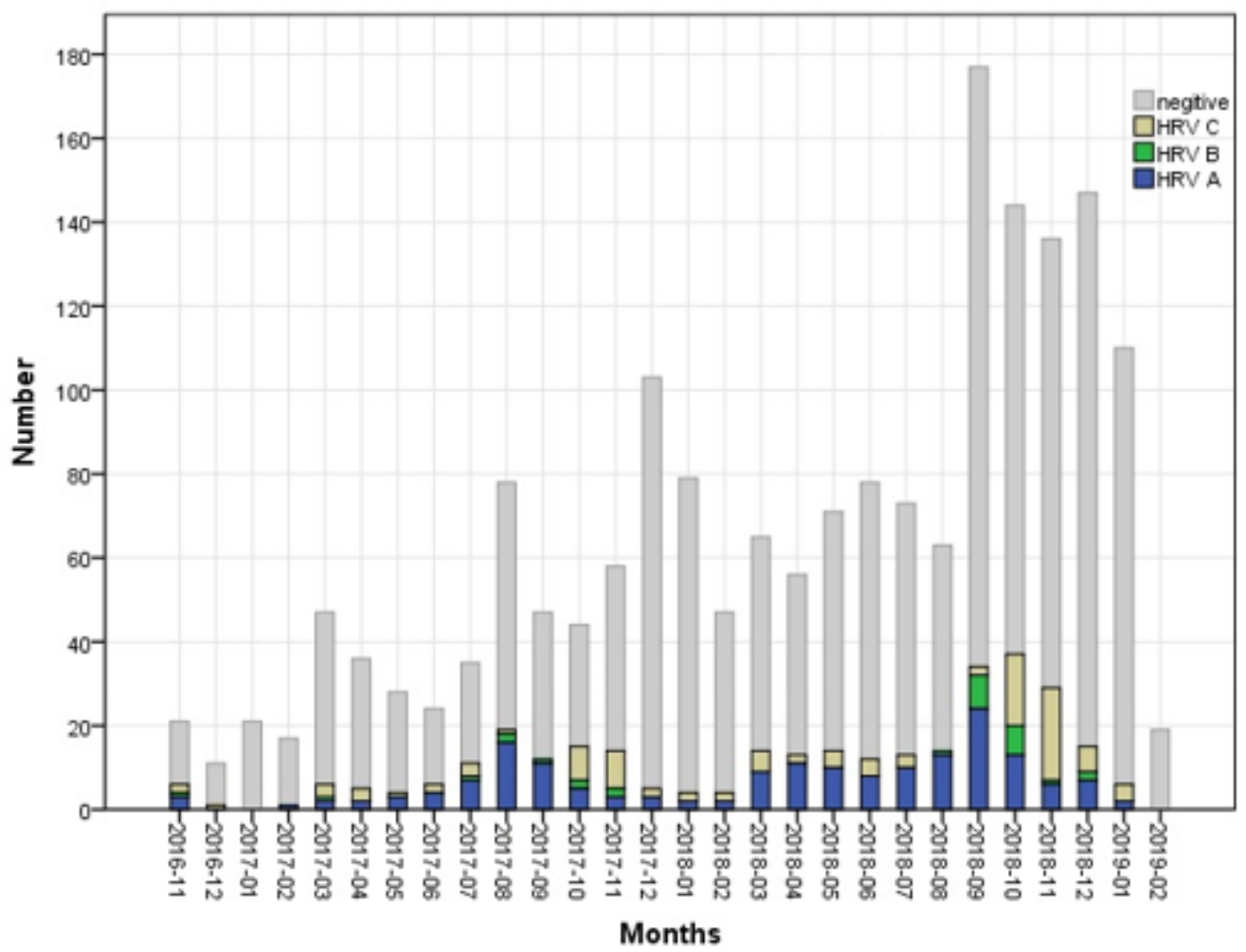

Figure 2

The monthly distribution of HRVs during Nov 2016 to Feb 2019 


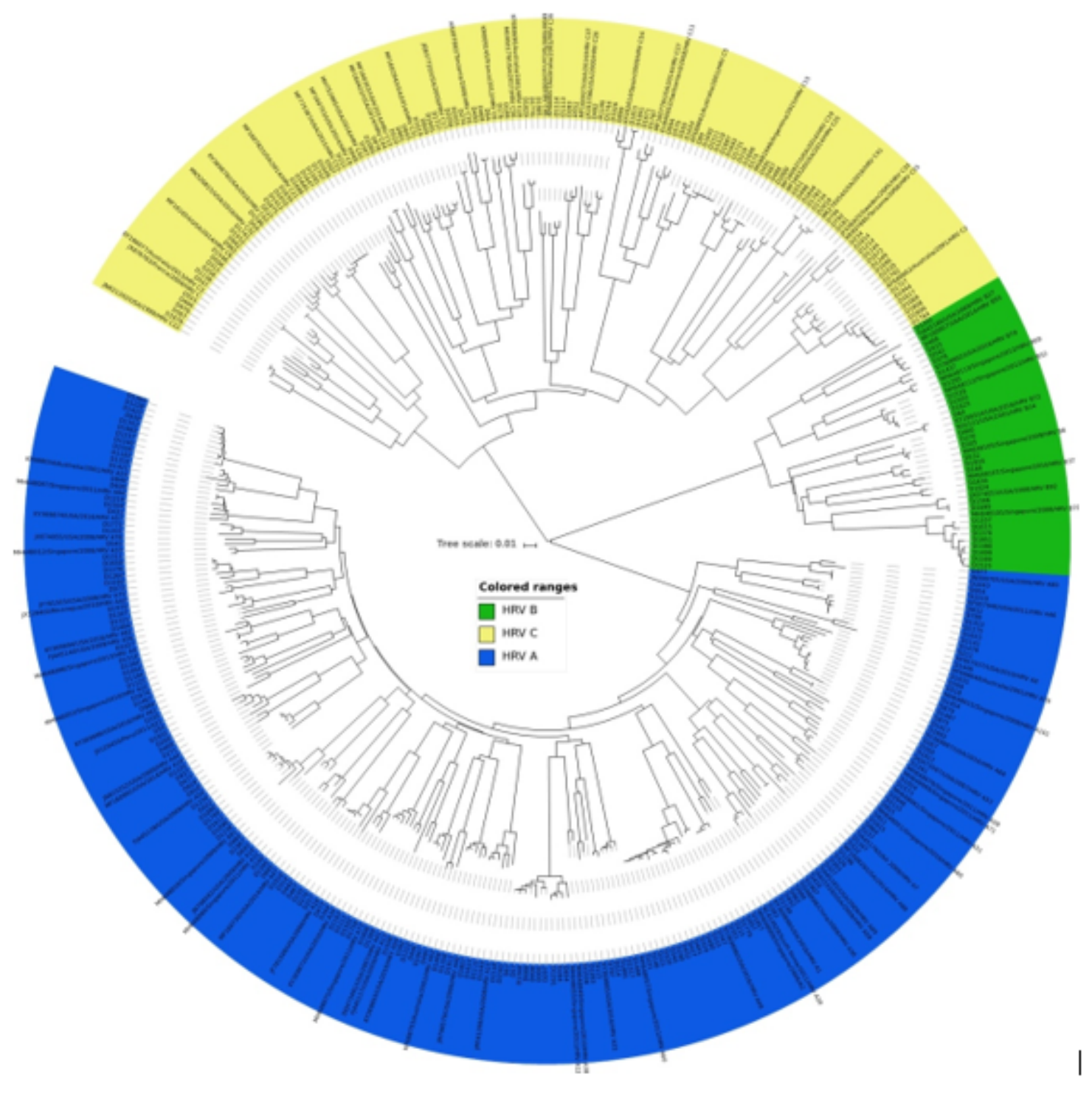

Figure 3

The phylogenetic tree constructed by MEGA version 6.0 software to identify the sero- or geno- types of HRV positive specimens 

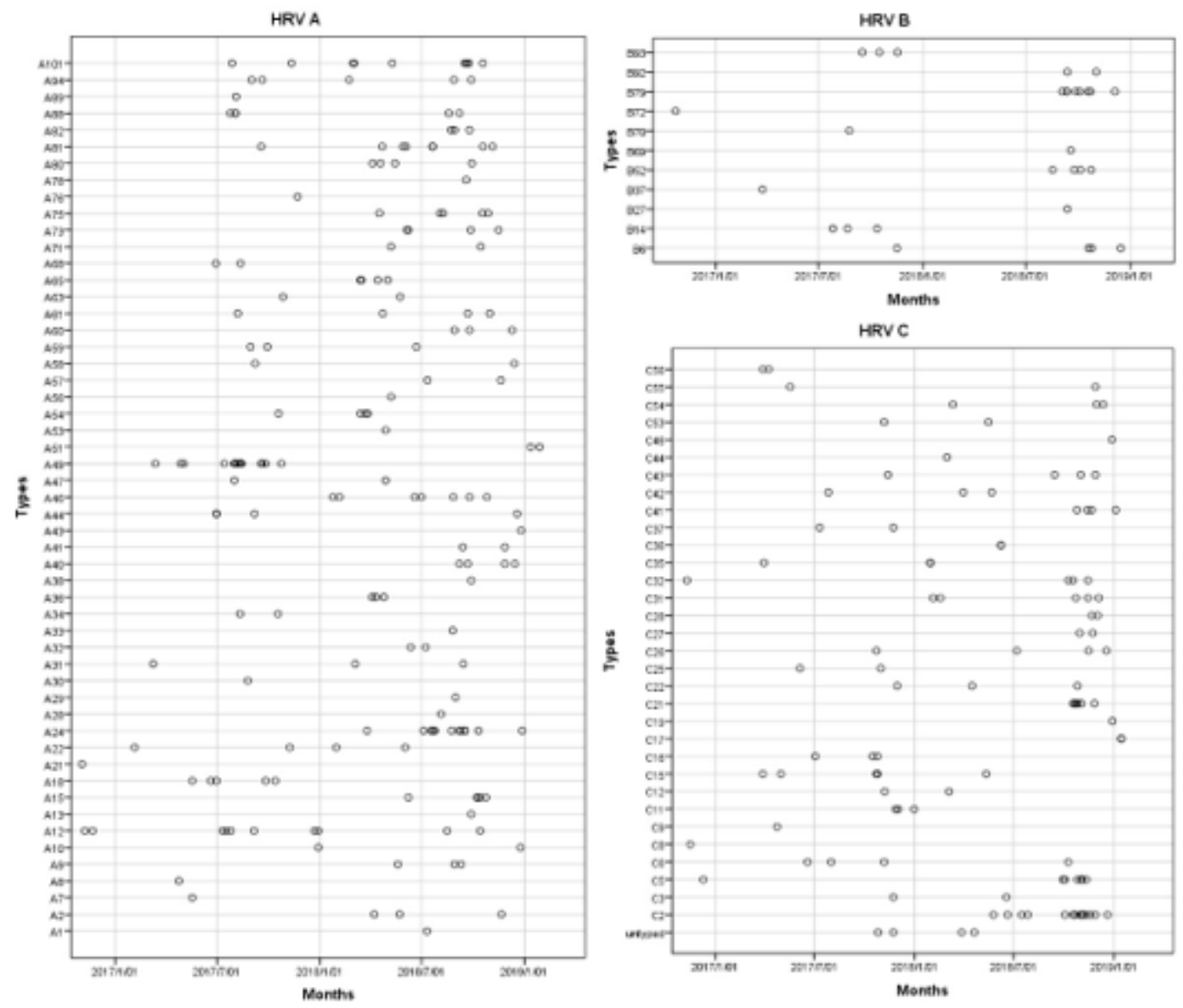

Figure 4

Scheme of the monthly distribution of HRV different sero- or geno- types 

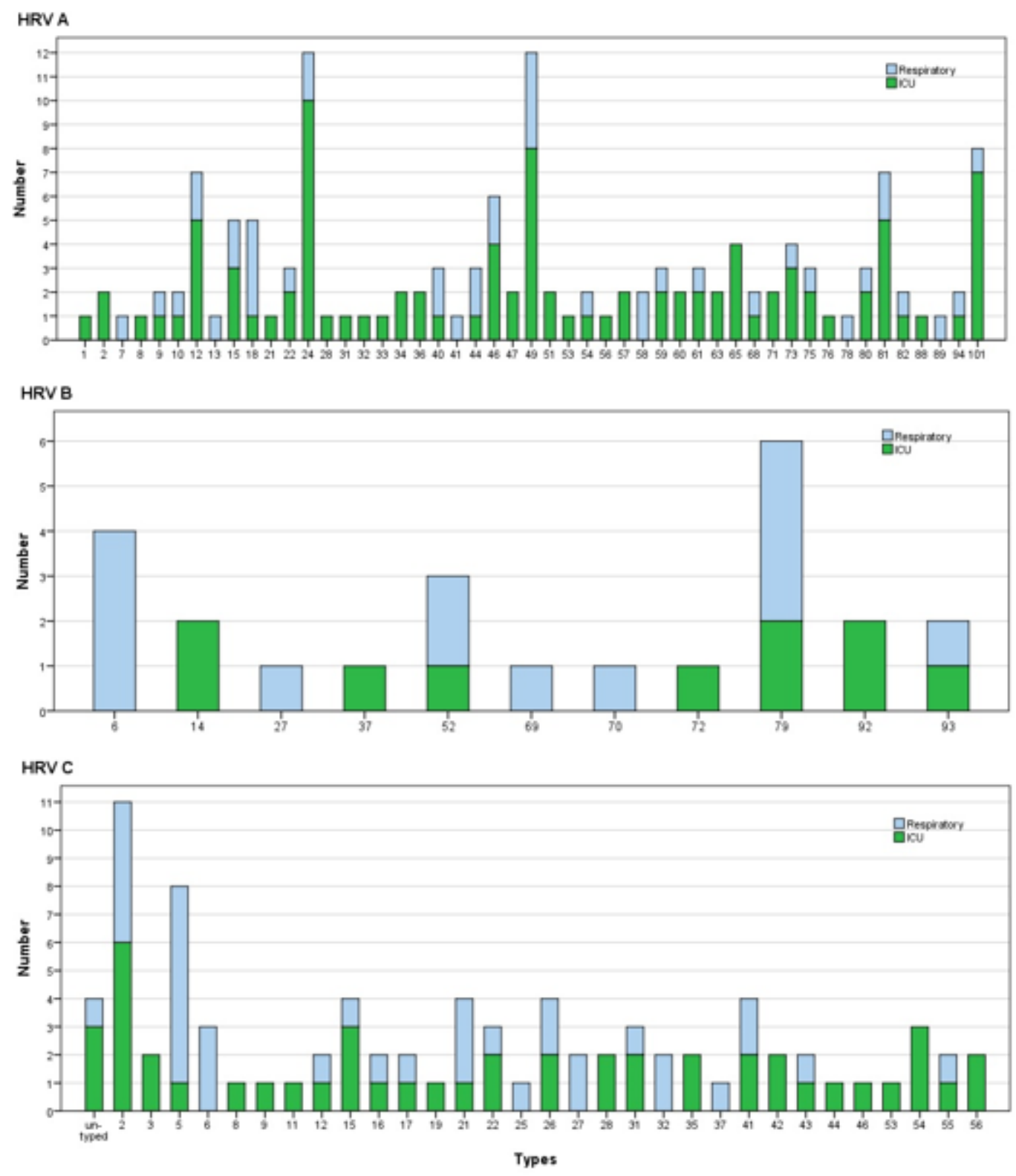

Figure 5

Distribution of sero- or geno-types of different HRV species in two groups: ICU group, Respiratory group 